\title{
IS THERE ASSOCIATION BETWEEN MIDKINE LEVELS AND PROGNOSIS OF COVID-19 DISEASE?
}

\author{
Ahmed Bilal Genc ${ }^{1}$, Selcuk Yaylaci ${ }^{2}$, Hamad Dheir ${ }^{2}$, Ahmed Cihad Genc ${ }^{1}$, Ilhan Yıldırım ${ }^{1}$, \\ Havva Kocayigit ${ }^{1}$, Fatma Betül Tuncer ${ }^{1}$, Hande Toptan $^{1}$, Erdem Çokluk ${ }^{1}$, Bekir Enes \\ Demiryurek $^{2}$, Ahmet Nalbant ${ }^{2}$, Tezcan Kaya ${ }^{2}$, Ali Tamer ${ }^{1}$, and Ö̈uz Karabay ${ }^{2}$ \\ ${ }^{1}$ Sakarya Universitesi \\ ${ }^{2}$ Sakarya University
}

February 8, 2021

\begin{abstract}
Aim: The aim of this study was to measure plasma midkine levels in patients with COVID-19 and to assess its clinical significance. Material and Method: 88 patients followed in our hospital with a diagnosis of COVID-19 were included in the study. Demographic characteristics, clinical and laboratory data of the patients were recorded, and the relationship between midkine level and prognosis and other parameters were investigated. Results: Of the 88 patients included in the study, 43 $(48.9 \%)$ were female and $45(51.1 \%)$ were male. $24(27 \%)$ of our cases were died. The mean age of non-survivals was $70 \pm 12.3$ and the survivals were $61.9 \pm 18.2$ years. Mortality predictors due to COVID-19 in group 2 patients were significantly higher than those in group $2(\mathrm{p}<0.05)$. The median (IR) value of the MK level was $152.5 \pm 125 \mathrm{pg} / \mathrm{ml}$ in all patients, $143 \pm 149 \mathrm{pg} / \mathrm{ml}$ in survivors and $165.5 \pm 76 \mathrm{pg} / \mathrm{ml}$ in those who died $(\mathrm{p}=0.546)$. The difference between these two groups compared according to mortality was not statistically significant The area under the ROC curve was found to be 0.542 (95\% CI $0.423-0.661, \mathrm{p}=$ 0.546). Conclusion: MK is not a biomarker that can reinforce known predictors of mortality in COVID-19 patients and can provide better predictions of mortality.
\end{abstract}

\section{Introduction}

The new coronavirus (SARS-COV-2) that causes COVID-19 disease was first detected in Wuhan, China in December 2019 and rapidly spread to more than 200 countries. $^{1}$ Although COVID-19 is generally characterized by respiratory system symptoms such as fever, sore throat, shortness of breath, and cough, cases presenting with atypical symptoms such as abdominal pain, headache, diarrhea, smell and taste disorders, musculoskeletal pain and skin lesions have been widely reported recently. ${ }^{2}$

Severe COVID-19 disease is an uncontrolled response of the immune system to infection, in which high levels of circulating cytokines lead to generalized inflammatory response with failure of at least one organ function and high mortality rates. ${ }^{3}$ Many COVID-19 related mortality biomarkers such as D-Dimer, C-reactive protein (CRP), Ferritin, pentraxin-3 and proinflammatory cytokines elevation have been demonstrated. ${ }^{4-10}$ However, the search for different disease-specific biomarkers continues.

Midkine (MK) is a multifunctional cytokine, expressed primarily in midgestation. It is a heparin-binding growth factor sensitive to retinoic acid released from various cell types during embryogenesis. Promotes angiogenesis, cell growth and cell migration. Midkine is also expressed in various malignancies, suggesting that it may play a role in tumorigenesis, perhaps through its effects on angiogenesis. Cytokines and growth factors are classified into structurally related protein families such as the fibroblast growth factor family. ${ }^{11}$ In addition, MK organizes the proliferation, differentiation, survival, adhesion, migration and gene expression of immune cells. At the same time, MK is a mitogenic, antiapoptotic, migrating, chemotactic, angiogenic 
and fibrinolytic molecule that plays a role in controlling the inflammatory response. ${ }^{12} \mathrm{MK}$ can alleviate hypoperfusion and myocardial cell damage due to hypoxia, as well as promote angiogenesis in myocardial infarction. ${ }^{13}$ However, it may accelerate tubular necrosis in drug or autoimmune kidney damage ${ }^{11}$ and pulmonary fibrosis due to acute respiratory distress syndrome (ARDS).$^{14}$ The interesting issue about MK is that it has been shown in studies that it has bactericidal, antifungal and antiviral effects in some viruses such as herpes simplex and human immunodeficiency virus (HIV). ${ }^{15-17}$

COVID-19 is a new disease and many proinflammatory cytokines and other acute phase proteins levels have been found to correlate with poor prognosis in the literature. However, there are no specific biomarkers in the prognosis and surveillance of COVID-19. ${ }^{18}$ The aim of our study was to investigate MK levels in COVID-19 infection and to explore whether there is a relationship between the prognosis of patients and midkine levels.

\section{Material and Methods:}

This study was conducted on 88 confirmed COVID-19 patients who were hospitalized due to symptomatic pneumonia between April 15, 2020 and August 15, 2020. The study was conducted in accordance with the Declaration of Helsinki, and after approval of the ethics committee of our university faculty of medicine (No:71522473/050.01.04/461). The study population was determined as patients hospitalized in the Training and Research Hospital within the specified period. Patients whose serum could be separated for midkine at admission to hospitalization were included in the study. Also, patients with symptomatic pneumonia, had indication for hospitalization and had confirmation of COVID-19 by reverse transcription-polymerase chain reaction (RT-PCR) from nasopharyngeal (NP) swabs were consecutively enrolled. The patients who did not have radiologic signs of pneumonia, NP RT-PCR negative, have malignancy, have confirmed bacterial infection at admission were excluded. The patients were divided into two groups according to surviving (Group 1=survive group and Group 2=non-survived patients). Both groups were compared according to demographic features, comorbid diseases and laboratory findings of patients. Before receiving any antimicrobial or anti-inflamatuar drug, the serum midkine was obtained from all patients at the first admission to the ward or intensive care unit (ICU).

\section{Statistical analysis}

Descriptive analyses were performed to provide information on general characteristics of the study population. Visual (probability plots, histograms) and analytical methods (Kolmogorov-Simirnov/Shapiro-Wilk's test) were used to determine whether they are normally distributed or not. Descriptive analyses were presented using medians and interquartile range (IR) for the non-normally distributed variables. The Mann-Whitney $\mathrm{U}$ Test was used for nonparametric tests, Independent Sample T Test was used for parametric tests to compare these parameters. Chi-square test used to compare the categorical variables between two groups. The categorical variables were presented as the frequency (\% percentage). The performance of midkine was assessed using receiver operating characteristic (ROC) curve analysis and by calculating the area under the curve (AUC) of the ROC curves. A p-value $<0.05$ was considered significant. Analyses were performed using SPSS statistical software (IBM SPSS Statistics, Version 22.0. Armonk, NY: IBM Corp.)

\section{Results}

The demographics of the 88 patients are provided in Table 1 . The cohort had a median age of $64,1 \pm 18,2$ years. There were $45(51,1 \%)$ men and $43(48,9 \%)$ women. The patients were divided into two groups according to their mortality as the survival group (group 1) and the non-survive group (group 2). Of the 88 patients included in the study, $24(27 \%)$ died. Ten of the female patients $(41.7 \%)$ and 14 of the male patients (58.3\%) resulted in death, and there was no significant difference in mortality between men and women $(\mathrm{p}=0.408)$. Mean age, the non-survive group was older than the survival group $(70 \pm 12.3$ years vs $61.9 \pm 18.2$ years, respectively; $\mathrm{p}=0.020$ ). With respect to diabetes mellitus, hypertension, heart disease, chronic obstructive pulmonary disease, chronic renal failure and other accompanying morbid diseases there was no significant difference between the two groups ( $\mathrm{p}>0.05) .27(42.2 \%)$ patients from group 1 have fever while it was present in only $4(16.7 \%)$ patients from group $2(\mathrm{p}=0.026)$. In addition, $23(35.9 \%)$ of the group 1 and 16 
$(66.7 \%)$ of the group 2 had shortness of breath $(\mathrm{p}=0.001)$. Other symptoms were not significantly different between both groups ( $\mathrm{p}>0.05$ ) (Figure 1). Mortality predictors due to COVID-19 in group 2 patients were significantly higher than those in group $2(\mathrm{p}<0.05)$ (Table 2$)$.

The median (IR) value of the MK level was $152.5 \mathrm{pg} / \mathrm{ml}$ (125) in all patients, $143 \mathrm{pg} / \mathrm{ml}$ (149) in survivors and $165.5 \mathrm{pg} / \mathrm{ml}(76)$ in those who died. However, the difference between these two groups compared according to mortality was not statistically significant $(\mathrm{p}=0.546)$ (Table 1$)$. ROC analysis was performed to determine whether there was a cut-off value that could be considered significant in predicting mortality in patients with Covid-19. The area under the ROC curve was found to be 0.542 (95\% Confidence Interval 0.423-0.661, $\mathrm{p}=$ 0.546). Therefore, a suitable cut-off value was not found for statistical significance (Figure 2).

Table 1: Demographic characteristics of patients with COVID-19

\begin{tabular}{|c|c|c|c|c|c|c|}
\hline & $\begin{array}{l}\text { Survivors } \\
\mathrm{n}=64\end{array}$ & $\begin{array}{l}\text { Non } \\
\text { Survivors } \\
\mathbf{n}=\mathbf{2 4}\end{array}$ & $\begin{array}{l}\text { Non } \\
\text { Survivors } \\
\mathrm{n}=\mathbf{2 4}\end{array}$ & $\begin{array}{l}\text { All } \\
\text { patients } \\
\mathrm{n}=88\end{array}$ & $\begin{array}{l}\text { All } \\
\text { patients } \\
\mathbf{n}=88\end{array}$ & p \\
\hline $\begin{array}{l}\text { Age, } \\
\text { year, } \\
\text { mean } \\
( \pm S D)\end{array}$ & $61,9(18,2)$ & $70(12,3)$ & $70(12,3)$ & $64,1(17,1)$ & $64,1(17,1)$ & $0,020^{*}$ \\
\hline $\begin{array}{l}\text { SEX } \\
\text { Female, } \\
\text { n (\%) }\end{array}$ & $33(51,6 \%)$ & $10(41,7 \%)$ & $10(41,7 \%)$ & $43(48,9 \%)$ & $43(48,9 \%)$ & $0,408^{* * *}$ \\
\hline $\begin{array}{l}\text { Diabetes } \\
\text { Mellitus, } \\
\text { n (\%) }\end{array}$ & $17(26,6 \%)$ & $7(29,2 \%)$ & $7(29,2 \%)$ & $24(27,3 \%)$ & $24(27,3 \%)$ & $0,807^{* * *}$ \\
\hline \multicolumn{2}{|c|}{$\begin{array}{l}\text { Hypertension,35 (54,7\%) } \\
\text { n (\%) }\end{array}$} & $13(54,2 \%)$ & $13(54,2 \%)$ & $48(54,5 \%)$ & $48(54,5 \%)$ & $0,965^{* * *}$ \\
\hline \multicolumn{7}{|l|}{$\begin{array}{l}\text { Disease, } \\
\text { n (\%) }\end{array}$} \\
\hline $\begin{array}{l}\text { COPD, } \\
(\%)\end{array}$ & $6(9,40 \%)$ & $3(12,5 \%)$ & $3(12,5 \%)$ & $9(10,2 \%)$ & $9(10,2 \%)$ & $0,700^{* * *}$ \\
\hline $\begin{array}{l}\text { CKD, n } \\
(\%)\end{array}$ & $5(7,80 \%)$ & $3(12,5 \%)$ & $3(12,5 \%)$ & $8(9,10 \%)$ & $8(9,10 \%)$ & $0,496^{* * *}$ \\
\hline \multicolumn{2}{|c|}{$\begin{array}{l}\text { Cerebrovascular } 4,70 \%) \\
\text { disease, } \\
\text { n (\%) }\end{array}$} & $4(17,4 \%)$ & $4(17,4 \%)$ & $7(8,00 \%)$ & $7(8,00 \%)$ & $0,076^{* * *}$ \\
\hline $\begin{array}{l}\text { Unit } \\
\text { Ward, n } \\
\text { (\%) }\end{array}$ & $47(73,4 \%)$ & $\begin{array}{l}0 \\
(0,00 \% \%)\end{array}$ & $\begin{array}{l}0 \\
(0,00 \% \%)\end{array}$ & $47(53,4 \%)$ & $47(53,4 \%)$ & $0,000 * * *$ \\
\hline $\begin{array}{l}\text { ICU, } \mathbf{n} \\
(\%)\end{array}$ & $17(26,6 \%)$ & $24(100, \%)$ & $24(100, \%)$ & $41(46,6 \%)$ & $41(46,6 \%)$ & \\
\hline
\end{tabular}




\begin{tabular}{|c|c|c|c|c|c|c|c|}
\hline SD:Standard & SD:Standard & SD:Standard & SD:Standard & SD:Standard & SD:Standard & SD:Standard & SD:Standard \\
\hline Deviation, & Deviation, & Deviation, & Deviation, & Deviation, & Deviation, & Deviation, & Deviation, \\
\hline IR: In- & IR: In- & IR: In- & IR: In- & IR: In- & IR: In- & IR: In- & IR: In- \\
\hline terquartile & terquartile & terquartile & terquartile & terquartile & terquartile & terquartile & terquartile \\
\hline Range, & Range, & Range, & Range, & Range, & Range, & Range, & Range, \\
\hline *:Indepen- & *:Indepen- & *:Indepen- & *:Indepen- & *:Indepen- & *:Indepen- & *:Indepen- & *:Indepen- \\
\hline Sample T & Sample T & Sample T & Sample T & Sample T & Sample T & Sample T & Sample T \\
\hline Test, **: & Test, **: & Test, **: & Test, **: & Test, **: & Test, **: & Test, **: & Test, **: \\
\hline Mann- & Mann- & Mann- & Mann- & Mann- & Mann- & Mann- & Mann- \\
\hline Whitney & Whitney & Whitney & Whitney & Whitney & Whitney & Whitney & Whitney \\
\hline $\begin{array}{l}\text { U Test, } \\
* * *: \text { Chi- }\end{array}$ & $\begin{array}{l}\text { U Test, } \\
* * * \text { :Chi- }\end{array}$ & $\begin{array}{l}\text { U Test, } \\
* * * \text { :Chi- }\end{array}$ & $\begin{array}{l}\text { U Test, } \\
* * * \text { :Chi- }\end{array}$ & $\begin{array}{l}\text { U Test, } \\
* * * \text { :Chi- }\end{array}$ & $\begin{array}{l}\text { U Test, } \\
* * *: \text { Chi- }\end{array}$ & $\begin{array}{l}\text { U Test, } \\
* * * \text { :Chi- }\end{array}$ & $\begin{array}{l}\text { U Test, } \\
* * * \text { :Chi- }\end{array}$ \\
\hline Square & Square & Square & Square & Square & Square & Square & Square \\
\hline Tests, & Tests, & Tests, & Tests, & Tests, & Tests, & Tests, & Tests, \\
\hline COPD: & COPD: & COPD: & COPD: & COPD: & COPD: & COPD: & COPD: \\
\hline Chronic & Chronic & Chronic & Chronic & Chronic & Chronic & Chronic & Chronic \\
\hline obstruc- & obstruc- & obstruc- & obstruc- & obstruc- & obstruc- & obstruc- & obstruc- \\
\hline tive & tive & tive & tive & tive & tive & tive & tive \\
\hline pulmonary & pulmonary & pulmonary & pulmonary & pulmonary & pulmonary & pulmonary & pulmonary \\
\hline disease, & disease, & disease, & disease, & disease, & disease, & disease, & disease, \\
\hline CKD: & CKD: & CKD: & CKD: & CKD: & CKD: & CKD: & CKD: \\
\hline chronic & chronic & chronic & chronic & chronic & chronic & chronic & chronic \\
\hline kidney & kidney & kidney & kidney & kidney & kidney & kidney & kidney \\
\hline disease, & disease, & disease, & disease, & disease, & disease, & disease, & disease, \\
\hline ICU: & ICU: & ICU: & ICU: & ICU: & ICU: & ICU: & ICU: \\
\hline intensive & intensive & intensive & intensive & intensive & intensive & intensive & intensive \\
\hline care unit & care unit & care unit & care unit & care unit & care unit & care unit & care unit \\
\hline
\end{tabular}

Table 2: Comparison of laboratory findings between patients with COVID-19

\begin{tabular}{|c|c|c|c|c|c|c|}
\hline & $\begin{array}{l}\text { Survivors } \\
\mathrm{n}=64\end{array}$ & $\begin{array}{l}\text { Non } \\
\text { Survivors } \\
\mathbf{n}=\mathbf{2 4}\end{array}$ & $\begin{array}{l}\text { Non } \\
\text { Survivors } \\
\mathbf{n}=\mathbf{2 4}\end{array}$ & $\begin{array}{l}\text { All } \\
\text { patients } \\
\mathbf{n}=88\end{array}$ & $\begin{array}{l}\text { All } \\
\text { patients } \\
\mathbf{n}=88\end{array}$ & $\mathbf{p}$ \\
\hline $\begin{array}{l}\text { WBC, } \\
\text { median } \\
(\mathrm{IR})\end{array}$ & $7(9,1)$ & $8,1(5,4)$ & $8,1(5,4)$ & $7,1(6,2)$ & $7,1(6,2)$ & $0,974^{* *}$ \\
\hline $\begin{array}{l}\text { Lymphocyte, } \\
\text { median } \\
\text { (IR) }\end{array}$ & $1,3(1,8)$ & $0,6(0,4)$ & $0,6(0,4)$ & $1,1(1,0)$ & $1,1(1,0)$ & $0,000^{* *}$ \\
\hline $\begin{array}{l}\text { Neutrophil, } \\
\text { median (IR) }\end{array}$ & $5(8,1)$ & $6,0(4,9)$ & $6,0(4,9)$ & $5,1(6,3)$ & $5,1(6,3)$ & $0,739^{* *}$ \\
\hline $\begin{array}{l}\text { Platelet, } \\
\text { median } \\
(\mathrm{IR})\end{array}$ & $\begin{array}{l}190,9 \\
(59,6)\end{array}$ & $\begin{array}{l}219,9 \\
(154,3)\end{array}$ & $\begin{array}{l}219,9 \\
(154,3)\end{array}$ & $\begin{array}{l}198,8 \\
(95,1)\end{array}$ & $\begin{array}{l}198,8 \\
(95,1)\end{array}$ & $0,862^{* *}$ \\
\hline $\begin{array}{l}\text { Prothrombin } \\
\text { time, } \\
\text { median } \\
\text { (IR) }\end{array}$ & $12,8(2,6)$ & $14,0(2,0)$ & $14,0(2,0)$ & $13,1(2,5)$ & $13,1(2,5)$ & $0,001^{* *}$ \\
\hline
\end{tabular}




\begin{tabular}{|c|c|c|c|c|c|c|}
\hline $\begin{array}{l}\text { D- } \\
\text { DIMER, } \\
\text { median } \\
\text { (IR) }\end{array}$ & $\begin{array}{l}1030,6 \\
(1287,2)\end{array}$ & $\begin{array}{l}3461,8 \\
(7462,5)\end{array}$ & $\begin{array}{l}3461,8 \\
(7462,5)\end{array}$ & $\begin{array}{l}1693,7 \\
(4136,2)\end{array}$ & $\begin{array}{l}1693,7 \\
(4136,2)\end{array}$ & $0,001 * *$ \\
\hline $\begin{array}{l}\text { Troponin, } \\
\text { median } \\
\text { (IR) }\end{array}$ & $28,8(75,0)$ & $\begin{array}{l}2272,8 \\
(10406,8)\end{array}$ & $\begin{array}{l}2272,8 \\
(10406,8)\end{array}$ & $\begin{array}{l}622,0 \\
(5357,2)\end{array}$ & $\begin{array}{l}622,0 \\
(5357,2)\end{array}$ & $0,000 * *$ \\
\hline $\begin{array}{l}\text { Ferritin, } \\
\text { median } \\
\text { (IR) }\end{array}$ & $\begin{array}{l}561,9 \\
(1003,7)\end{array}$ & $\begin{array}{l}1195,1 \\
(1770,8)\end{array}$ & $\begin{array}{l}1195,1 \\
(1770,8)\end{array}$ & $\begin{array}{l}736,6 \\
(1282,9)\end{array}$ & $\begin{array}{l}736,6 \\
(1282,9)\end{array}$ & $0,004^{* *}$ \\
\hline $\begin{array}{l}\text { Serum } \\
\text { albumin, } \\
\text { mean } \\
( \pm \mathrm{SD})\end{array}$ & $3,4(0,5)$ & $3,0(0,4)$ & $3,0(0,4)$ & $3,3(0,5)$ & $3,3(0,5)$ & $0,000^{*}$ \\
\hline $\begin{array}{l}\text { LDH, } \\
\text { median } \\
\text { (IR) }\end{array}$ & $\begin{array}{l}331,4 \\
(155,4)\end{array}$ & $\begin{array}{l}499,3 \\
(182,8)\end{array}$ & $\begin{array}{l}499,3 \\
(182,8)\end{array}$ & $\begin{array}{l}375,8 \\
(178,3)\end{array}$ & $\begin{array}{l}375,8 \\
(178,3)\end{array}$ & $0,000^{* *}$ \\
\hline $\begin{array}{l}\text { CRP, } \\
\text { median } \\
\text { (IR) }\end{array}$ & $\begin{array}{l}33,5 \\
(131,0)\end{array}$ & $\begin{array}{l}161,5 \\
(41,0)\end{array}$ & $\begin{array}{l}161,5 \\
(41,0)\end{array}$ & $\begin{array}{l}72,0 \\
(153,5)\end{array}$ & $\begin{array}{l}72,0 \\
(153,5)\end{array}$ & $0,001^{* *}$ \\
\hline $\begin{array}{l}\text { Procalcitonin, } \\
\text { median } \\
\text { (IR) }\end{array}$ & $0,1(0,2)$ & $0,3(0,6)$ & $0,3(0,6)$ & $0,1(0,3)$ & $0,1(0,3)$ & $0,000 * *$ \\
\hline $\begin{array}{l}\text { Fibrinojen, } \\
\text { median } \\
\text { (IR) }\end{array}$ & $\begin{array}{l}377,8 \\
(105,0)\end{array}$ & $\begin{array}{l}395,8 \\
(87,5)\end{array}$ & $\begin{array}{l}395,8 \\
(87,5)\end{array}$ & $\begin{array}{l}383,1 \\
(99,9)\end{array}$ & $\begin{array}{l}383,1 \\
(99,9)\end{array}$ & $0,06^{* *}$ \\
\hline $\begin{array}{l}\text { Laktat, } \\
\text { median } \\
\text { (IR) }\end{array}$ & $1,6(0,7)$ & $2,1(0,7)$ & $2,1(0,7)$ & $1,7(0,9)$ & $1,7(0,9)$ & $0,001 * *$ \\
\hline $\begin{array}{l}\text { Midkine, } \\
\text { median }\end{array}$ & $\begin{array}{l}143 \\
(149,0)\end{array}$ & $\begin{array}{l}143 \\
(149,0)\end{array}$ & $\begin{array}{l}165,5 \\
(76,0)\end{array}$ & $\begin{array}{l}165,5 \\
(76,0)\end{array}$ & $\begin{array}{l}152,5 \\
(125,0)\end{array}$ & $0,546^{* *}$ \\
\hline
\end{tabular}




\begin{tabular}{|c|c|c|c|c|c|c|c|}
\hline SD:Standard & SD:Standard & SD:Standard & SD:Standard & SD:Standard & SD:Standard & SD:Standard & SD:Standard \\
\hline Deviation, & Deviation, & Deviation, & Deviation, & Deviation, & Deviation, & Deviation, & Deviation, \\
\hline $\begin{array}{l}\text { IR: In- } \\
\text { terquartile }\end{array}$ & $\begin{array}{l}\text { IR: In- } \\
\text { terquartile }\end{array}$ & $\begin{array}{l}\text { IR: In- } \\
\text { terquartile }\end{array}$ & $\begin{array}{l}\text { IR: In- } \\
\text { terquartile }\end{array}$ & $\begin{array}{l}\text { IR: In- } \\
\text { terquartile }\end{array}$ & $\begin{array}{l}\text { IR: In- } \\
\text { terquartile }\end{array}$ & $\begin{array}{l}\text { IR: In- } \\
\text { terquartile }\end{array}$ & $\begin{array}{l}\text { IR: In- } \\
\text { terquartile }\end{array}$ \\
\hline Range, & Range, & Range, & Range, & Range, & Range, & Range, & Range, \\
\hline $\begin{array}{l}\text { *:Indepen- } \\
\text { dent }\end{array}$ & $\begin{array}{l}\text { *:Indepen- } \\
\text { dent }\end{array}$ & $\begin{array}{l}\text { *:Indepen- } \\
\text { dent }\end{array}$ & $\begin{array}{l}\text { *:Indepen- } \\
\text { dent }\end{array}$ & $\begin{array}{l}\text { *:Indepen- } \\
\text { dent }\end{array}$ & $\begin{array}{l}\text { *:Indepen- } \\
\text { dent }\end{array}$ & $\begin{array}{l}\text { *:Indepen- } \\
\text { dent }\end{array}$ & $\begin{array}{l}\text { *:Indepen- } \\
\text { dent }\end{array}$ \\
\hline Sample T & Sample T & Sample T & Sample T & Sample T & Sample T & Sample T & Sample T \\
\hline Test, **: & Test, **: & Test, $* *:$ & Test, **: & Test, **: & Test, $* *:$ & Test, **: & Test, **: \\
\hline Mann- & Mann- & Mann- & Mann- & Mann- & Mann- & Mann- & Mann- \\
\hline Whitney & Whitney & Whitney & Whitney & Whitney & Whitney & Whitney & Whitney \\
\hline $\begin{array}{l}\text { U Test, } \\
* * *: \text { Chi- }\end{array}$ & $\begin{array}{l}\text { U Test, } \\
* * *: \text { Chi- }\end{array}$ & $\begin{array}{l}\text { U Test, } \\
* * * \text { :Chi- }\end{array}$ & $\begin{array}{l}\text { U Test, } \\
* * *: \text { Chi- }\end{array}$ & $\begin{array}{l}\text { U Test, } \\
* * * \text { :Chi- }\end{array}$ & $\begin{array}{l}\text { U Test, } \\
* * *: \text { Chi- }\end{array}$ & $\begin{array}{l}\text { U Test, } \\
* * * \text { :Chi- }\end{array}$ & $\begin{array}{l}\text { U Test, } \\
* * *: \text { Chi- }\end{array}$ \\
\hline Square & Square & Square & Square & Square & Square & Square & Square \\
\hline Tests, & Tests, & Tests, & Tests, & Tests, & Tests, & Tests, & Tests, \\
\hline WBC: & WBC: & WBC: & WBC: & WBC: & WBC: & WBC: & WBC: \\
\hline white & white & white & white & white & white & white & white \\
\hline blood & blood & blood & blood & blood & blood & blood & blood \\
\hline cells, & cells, & cells, & cells, & cells, & cells, & cells, & cells, \\
\hline LDH: & LDH: & LDH: & LDH: & LDH: & LDH: & LDH: & LDH: \\
\hline lactate & lactate & lactate & lactate & lactate & lactate & lactate & lactate \\
\hline dehydro- & dehydro- & dehydro- & dehydro- & dehydro- & dehydro- & dehydro- & dehydro- \\
\hline genase, & genase, & genase, & genase, & genase, & genase, & genase, & genase, \\
\hline CRP: & CRP: & CRP: & CRP: & CRP: & CRP: & CRP: & CRP: \\
\hline $\begin{array}{l}\text { C-reactive } \\
\text { protein }\end{array}$ & $\begin{array}{l}\text { C-reactive } \\
\text { protein }\end{array}$ & $\begin{array}{l}\text { C-reactive } \\
\text { protein }\end{array}$ & $\begin{array}{l}\text { C-reactive } \\
\text { protein }\end{array}$ & $\begin{array}{l}\text { C-reactive } \\
\text { protein }\end{array}$ & $\begin{array}{l}\text { C-reactive } \\
\text { protein }\end{array}$ & $\begin{array}{l}\text { C-reactive } \\
\text { protein }\end{array}$ & $\begin{array}{l}\text { C-reactive } \\
\text { protein }\end{array}$ \\
\hline
\end{tabular}

$80,00 \%$

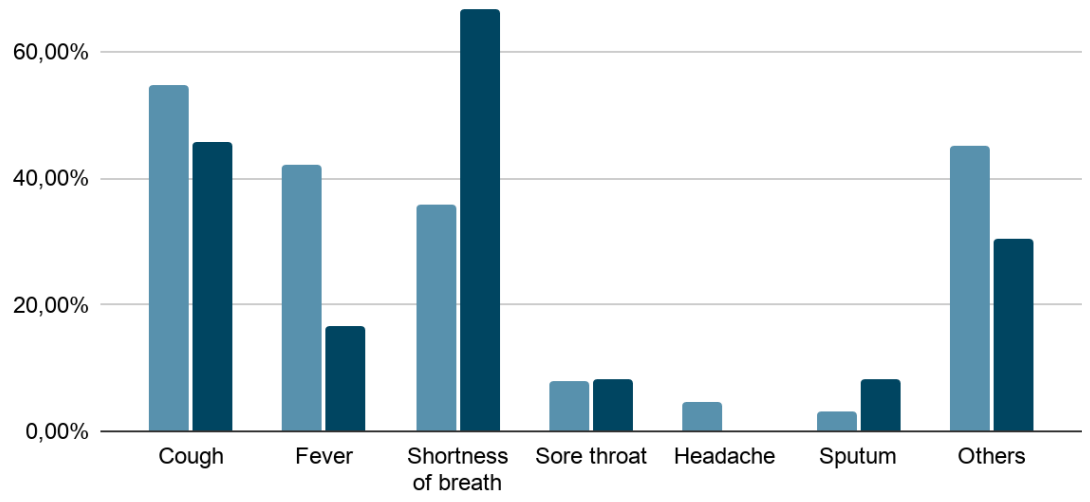

Figure 1: Complaints of patients with COVID-19 at admission 


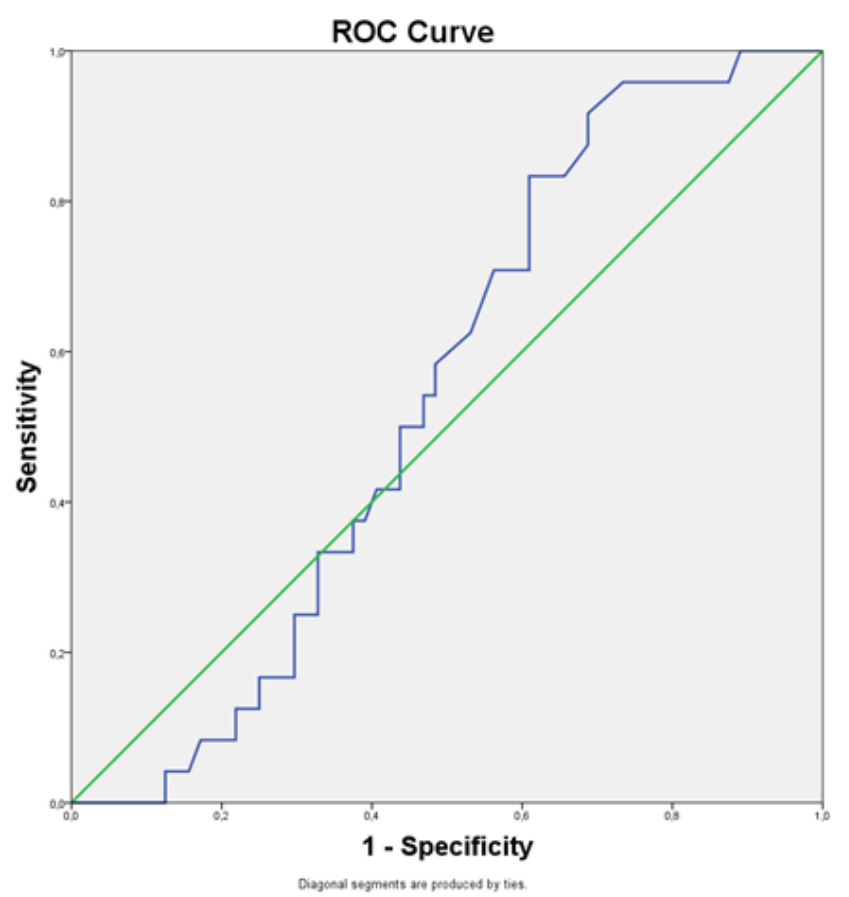

Figure 2: ROC curves of Midkine on differentiating mortal patients from survival patients with COVID-19. ROC: Receiver operating characteristic curve

\section{Discussion}

In the present study, to our knowledge, for the first time we have measured MK levels in consecutive COVID19 patients. There was no significant difference found between mortal and survival groups with respect to serum MK levels in followed patients. At the same time, it was determined that serum MK value did not have a significant importance in predicting mortality due to COVID-19 disease. Since COVID-19 disease mainly affects respiratory and immune systems, respiratory epithelial cells and immune $\mathrm{T}$ lymphocytes appear to be clear targets for COVID-19 disease. ${ }^{15-19}$ MK occurs in varying concentrations in the skin and major respiratory tract, against potential pathogens which the body encounters for the first time. This protein is a growth factor which its function has been investigated in many healthy volunteers, some bacterial or viral infections and serious conditions such as sepsis. ${ }^{15,16,20,21}$ The serum levels of MK in healthy individuals are ranged from 302 to $1068 \mathrm{pg} / \mathrm{mL} .{ }^{20}$ In the in vitro studies, the researchers found that MK had a strong bactericidal activity against the respiratory pathogen Streptococcus pneumoniae and Escherichia coli but no activity against Staphylococcus aureus. ${ }^{22,23}$ Also, it's a cytokine that inhibits HIV infection in an autocrine and paracrine manner by preventing the adherence of HIV particles when added to CD4 cells before HIV virus, but it has no significant effect when added after virus entry to the $\mathrm{T}$ lymphocyte. ${ }^{17}$ In our study population, we measured MK values within normal limits in mortal and non-mortal patients. A significant sensitivity and specificity could not be reached as a result of the ROC analysis performed to establish a certain cut-off value. These results may indicate that COVID-19 disease suggests that unlike some viruses, it can enter the cell without using MK protein and causes immunopathological damage. However, this data needs to be verified with different methods.

Recent studies showed that MK has a new role in acute and chronic inflammatory diseases including colitis, atherosclerosis, multiple sclerosis, nephritis, and rheumatoid arthritis have been shown to be alleviated in the absence of MK in animal models. ${ }^{24}$ These chronic inflamatuar diseases critically affect patients' quality of life. For instance; in cases of atherosclerosis, when endothelial dysfunction develops, MK excessively 
expresses and causes leukocyte infiltration in the damaged area. In animal study, it has been shown that MK-deficient mice did not increase leukocyte infiltration. ${ }^{25}$ It has been shown to cause fatal thrombotic microangiopathies as a result of hyperinflammation occurring secondary to COVID-19 disease. COVID-19 virus invading the endothelium with its ACE2 receptor causes severe endothelial damage and degradation in endothelial cell membranes. ${ }^{26,27}$ Despite this, although our study population accompanied chronic diseases such as diabetes mellitus and hypertension, we did not find any significant relationship between MK levels and mortality. Perhaps, MK's may have a more meaningful function in the chronic inflammation process rather than in acute inflammation. Also, we investigated the levels of MK only as soon as patients were hospitalized. Maybe if we had taken several MK measurements at different stages of the disease during hospitalization, we would have reached a more accurate result.

Many acute phase reactants and proinflammatory cytokines have been identified as determining factors in mortality from COVID-19 disease. ${ }^{18}$ In our study, we think that the increased serum values of these indicators of mortality in COVID-19 patients was in accordance with the literature supporting the reliability of the patient population and the results of the study for MK.

A small number of patients and the shorter observation period are among the limitations of our study. In addition, plasma MK levels were measured only once at admission and were not continuously monitored; therefore, trends in plasma MK levels in survivors and non-survivors are unknown. We think that MK should be studied in subgroups such as patients with bacterial superinfection.

In conclusion, MK is not a biomarker that can reinforce known predictors of mortality in COVID-19 patients and can provide better predictions of mortality. Nethertheless, COVID-19 is a self-limited infection, in which the strength of the host's immune strength plays a significant role against it in the common patients [28]. It can be said that the MK can help this self-limited infection due to its antiviral feature. This assumption should be confirmed by in vitro and in vivo studies of COVID-19.

Acknowledgments: We would like to thank the Sakarya Training and Research Hospital and the Infection Control Committee for their support in this research.

Conflict of Interest: The authors declare that they have no conflict of interest.

Funding information: This work has not received any funding

\section{References}

1. Guan W-J, Ni Z-Y, Hu Y, Liang W-H, Ou C-Q, He J-X, et al. Clinical Characteristics of Coronavirus Disease 2019 in China. N Engl J Med. 2020;382: 1708-1720.

2. Karadaş Ö, Öztürk B, Sonkaya AR. A prospective clinical study of detailed neurological manifestations in patients with COVID-19. Neurol Sci. 2020;41: 1991-1995.

3. Wu T, Zuo Z, Kang S, Jiang L, Luo X, Xia Z, et al. Multi-organ Dysfunction in Patients with COVID-19: A Systematic Review and Meta-analysis. Aging Dis. 2020;11: 874-894.

4. Medetalibeyoglu A, Emet S, Kose M, Akpinar TS, Senkal N, Catma Y, et al. Serum Endocan Levels on Admission Are Associated With Worse Clinical Outcomes in COVID-19 Patients: A Pilot Study. Angiology. 2020; 3319720961267.

5. GenÇ AB, Yaylaci S, Dheİr H, GenÇ AC, İşsever K, ÇekİÇ D, et al. The predictive and diagnostic accuracy of long Pentraxin-3 in COVID-19 Pneumonia. Turk J Med Sci. 2020. doi:10.3906/sag-2011-32

6. Characteristics and Outcomes of Critically Ill Patients with Covid-19 in Sakarya, Turkey: A Single Center Cohort Study. TURKISH JOURNAL OF MEDICAL SCIENCES. 2020. doi:10.3906/sag-2005-57

7. Nalbant A, Kaya T, Varim C, Yaylaci S, Tamer A, Cinemre H. Can the neutrophil/lymphocyte ratio (NLR) have a role in the diagnosis of coronavirus 2019 disease (COVID-19)? Rev Assoc Med Bras. 2020;66: $746-751$. 
8. Varim C, Yaylaci S, Demirci T, Kaya T, Nalbant A, Dheir H, et al. Neutrophil count to albumin ratio as a new predictor of mortality in patients with COVID-19 infection. Rev Assoc Med Bras. 2020;66Suppl 2: $77-81$.

9. Clinical course of COVID-19 disease in immunosuppressed renal transplant patients. TURKISH JOURNAL OF MEDICAL SCIENCES. 2020. doi:10.3906/sag-2007-260

10. Ponti G, Maccaferri M, Ruini C, Tomasi A, Ozben T. Biomarkers associated with COVID19 disease progression. Critical Reviews in Clinical Laboratory Sciences. 2020. pp. 389-399. doi:10.1080/10408363.2020.1770685

11. Muramatsu T, Kadomatsu K. Midkine: an emerging target of drug development for treatment of multiple diseases. Br J Pharmacol. 2014;171: 811-813.

12. Yazihan N. Midkine in inflammatory and toxic conditions. Curr Drug Deliv. 2013;10: 54-57.

13. Horiba M, Kadomatsu K, Yasui K, Lee J-K, Takenaka H, Sumida A, et al. Midkine Plays a Protective Role Against Cardiac Ischemia/Reperfusion Injury Through a Reduction of Apoptotic Reaction. Circulation. 2006. pp. 1713-1720. doi:10.1161/circulationaha.106.632273

14. Zhang R, Pan Y, Fanelli V, Wu S, Luo AA, Islam D, et al. Mechanical Stress and the Induction of Lung Fibrosis via the Midkine Signaling Pathway. Am J Respir Crit Care Med. 2015;192: 315-323.

15. Nordin SL, Andersson C, Bjermer L, Bjartell A, Mörgelin M, Egesten A. Midkine is part of the antibacterial activity released at the surface of differentiated bronchial epithelial cells. J Innate Immun. 2013;5: $519-530$.

16. Ostrander M, Fingar H, Seddon A, Böhlen P, Backer J. Anti-viral activity of human recombinant heparinbinding proteins HBNF and MK. Biochem Biophys Res Commun. 1992;189: 1189-1195.

17. Hovanessian AG. Midkine, a cytokine that inhibits HIV infection by binding to the cell surface expressed nucleolin. Cell Res. 2006;16: 174-181.

18. Xu Z, Shi L, Wang Y, Zhang J, Huang L, Zhang C, et al. Pathological findings of COVID-19 associated with acute respiratory distress syndrome. The Lancet Respiratory Medicine. 2020. pp. 420-422. doi:10.1016/s2213-2600(20)30076-x

19. Fung S-Y, Yuen K-S, Ye Z-W, Chan C-P, Jin D-Y. A tug-of-war between severe acute respiratory syndrome coronavirus 2 and host antiviral defence: lessons from other pathogenic viruses. Emerging Microbes \& Infections. 2020. pp. 558-570. doi:10.1080/22221751.2020.1736644

20. Ibusuki M, Fujimori H, Yamamoto Y, Ota K, Ueda M, Shinriki S, et al. Midkine in plasma as a novel breast cancer marker. Cancer Sci. 2009;100: 1735-1739.

21. Krzystek-Korpacka M, Mierzchala M, Neubauer K, Durek G, Gamian A. Midkine, a multifunctional cytokine, in patients with severe sepsis and septic shock: a pilot study. Shock. 2011;35: 471-477.

22. Muramatsu T. Midkine and Pleiotrophin: Two Related Proteins Involved in Development, Survival, Inflammation and Tumorigenesis. Journal of Biochemistry. 2002. pp. 359-371. doi:10.1093/oxfordjournals.jbchem.a003231

23. Svensson SL, Pasupuleti M, Walse B, Malmsten M, Mörgelin M, Sjögren C, et al. Midkine and Pleiotrophin Have Bactericidal Properties. Journal of Biological Chemistry. 2010. pp. 16105-16115. doi:10.1074/jbc.m109.081232

24. Weckbach LT, Muramatsu T, Walzog B. Midkine in Inflammation. The Scientific World JOURNAL. 2011. pp. 2491-2505. doi: $10.1100 / 2011 / 517152$ 
25. Horiba M, Kadomatsu K, Nakamura E, Muramatsu H, Ikematsu S, Sakuma S, et al. Neointima formation in a restenosis model is suppressed in midkine-deficient mice. Journal of Clinical Investigation. 2000. pp. 489495. doi: $10.1172 /$ jci7208

26. Zhu N, Zhang D, Wang W, Li X, Yang B, Song J, et al. A Novel Coronavirus from Patients with Pneumonia in China, 2019. New England Journal of Medicine. 2020. pp. 727-733. doi:10.1056/nejmoa2001017

27. Varga Z, Flammer AJ, Steiger P, Haberecker M, Andermatt R, Zinkernagel AS, et al. Endothelial cell infection and endotheliitis in COVID-19. The Lancet. 2020. pp. 1417-1418. doi:10.1016/s0140-6736(20)309375

28. Cascella M, Rajnik M, Cuomo A, Dulebohn SC, Di Napoli R. Features, Evaluation, and Treatment of Coronavirus. StatPearls. Treasure Island (FL): StatPearls Publishing; 2020. 